\title{
LA LUMIĖRE, IMAGE DE DIEU ET NOM DE L'HOMME CHEZ GRÉGOIRE DE NAZIANZE
}

\author{
Guillaume Bady
}

Vrin | «Revue des sciences philosophiques et théologiques »

2013/4 Tome 97 | pages 459 à 476

ISSN 0035-2209

Article disponible en ligne à l'adresse :

https://www.cairn.info/revue-des-sciences-philosophiques-ettheologiques-2013-4-page-459.htm

Distribution électronique Cairn.info pour Vrin.

(C) Vrin. Tous droits réservés pour tous pays.

La reproduction ou représentation de cet article, notamment par photocopie, n'est autorisée que dans les limites des conditions générales d'utilisation du site ou, le cas échéant, des conditions générales de la licence souscrite par votre établissement. Toute autre reproduction ou représentation, en tout ou partie, sous quelque forme et de quelque manière que ce soit, est interdite sauf accord préalable et écrit de l'éditeur, en dehors des cas prévus par la législation en vigueur en France. Il est précisé que son stockage dans une base de données est également interdit. 


\title{
LA LUMIÈRE, IMAGE DE DIEU ET NOM DE L'HOMME CHEZ GRÉGOIRE DE NAZIANZE*
}

\author{
par Guillaume BADY \\ Institut Catholique de Paris - CNRS (HiSoMA)
}

《Lumière née de la lumière » : l'expression est dite régulièrement à propos du Christ par des millions de chrétiens. Pourquoi cette expression ${ }^{1}$ ? Était-elle nécessaire? De fait, elle est absente dans certaines sources ${ }^{2}$, dont la citation du symbole de Nicée lors de la $5^{\mathrm{e}}$ session du concile de Chalcédoine en 451.

Pourtant ces quelques mots, à en croire Joseph Moingt ${ }^{3}$, auraient marqué rien moins qu'un « tournant de civilisation » :

L'analogie nicéenne de la «lumière [issue] de la lumière » (alors que la Bible hébraïque n'applique pas à Yahvé le nom de Lumière) pourrait également être une marque de l'inculturation du christianisme aux religions solaires du bassin méditerranéen; les noms de la divinité païenne bientôt décernés au Christ : Pantocratôr, Dies (transcription latine de Zeus), Sol invictus en sont d'autres indices. Un tournant

* Les prémices de cet article ont été présentées lors de la journée d'études « Lumières de l'Antiquité » à l'Institut Catholique de Paris le 8 avril 2011.

1. Les commentaires modernes du symbole de Nicée privilégient bien entendu le mot «consubstantiel»; voir tout de même Ignacio OrTIZ DE URBINA, El Símbolo niceno, Madrid, CSIC, 1947, p. 140-151; Éphrem BoUlarand, L'Hérésie d'Arius et la «foi » de Nicée, t. 2, Paris, Letouzey \& Ané, 1972, p. 308-321; Reinhard StaATs, Das Glaubensbekenntnis von Nizäa-Konstantinopel. Historische und theologische Grundlagen, Darmstadt, Wissenschaftliche Buchgesellschaft, 1996, p. 227 et 231-234.

2. Giuseppe Dossetti, Il simbolo di Nicea e di Costantinopoli, Rome, Herder, 1967, p. 228-229 (symbole de Nicée), 244-245 (symbole de Constantinople).

3. Joseph Moingt, L’Homme qui venait de Dieu, Paris, Éd. du Cerf (coll. « Cogitatio fidei » 176), 1993, p. 167. 
considérable de civilisation est amorcé. S'il était justifié de parler du christianisme ancien en termes de « judéo-christianisme », cela ne l'est plus à partir de Nicée.

Quoi qu'il en soit du virage culturel dont l'expression « lumière née de la lumière » serait la trace ${ }^{4}$, l'origine de la formule, du moins, n'est pas complètement obscure. Au concile de Nicée en 325, Eusèbe de Césarée dit avoir proposé le symbole de foi de son Église où figurait l'expression ; dès lors celle-ci serait passée dans le symbole de Nicée, puis dans ce qu'on appelle par commodité le symbole de NicéeConstantinople.

L'expression elle-même est attestée dès la fin du II $^{\mathrm{e}}$ siècle chez Tertullien et peu après sans doute chez Hippolyte. Dans le Contre Noët attribué à ce dernier ${ }^{5}$, on lit en effet que Dieu « engendre la lumière (à

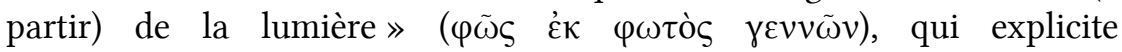
parfaitement les trois petits mots du symbole. L'Apologétique de Tertullien ${ }^{6}$ permet quant à elle de trouver, sans doute, la raison de cette image :

Quand un rayon est lancé hors du soleil, c'est une partie qui part du tout ; mais le soleil est dans le rayon, parce que c'est un rayon du soleil, et que la substance n'est pas divisée, mais étendue, comme la lumière qui s'allume à la lumière (ut lumen de lumine accensum). La matière-source demeure entière et ne perd rien, même si elle communique sa nature par plusieurs canaux. Ainsi, ce qui est sorti de Dieu est Dieu, Fils de Dieu, et les deux ne font qu'un.

En plus de ses multiples fondements scripturaires dans la Septante et surtout dans le Nouveau Testament, l'expression «lumière née de la lumière » présentait l'avantage de connoter l'identité de nature du Père et du Fils ${ }^{7}$, malgré la méfiance d'Arius vis-à-vis de cette image ${ }^{8}$.

4. L'assertion de J. Moingt se veut sans doute plus suggestive que démonstrative ; parmi les réserves que l'on peut formuler, il y aurait par exemple le caractère tardif de la date de 325 pour marquer l'inculturation au paganisme, ainsi que la trop grande distinction entre Bible hébraïque et Bible grecque, toutes deux étant issues du judaïsme, $y$ compris, bien sûr, le Nouveau Testament dans sa continuité avec l'Ancien. Il reste vrai, plus généralement, que le début de l'ère constantinienne opère un tournant majeur du christianisme, en particulier vers la romanité.

5. Hippolyte, Contre Noët, édité et traduit par Pierre NAutin, Hippolyte. Contre les hérésies. Fragment. Étude et édition critique, Paris, Éd. du Cerf (coll. «Études et textes pour l'histoire du dogme de la Trinité »2), 1949, p. 253, lignes 5 et 10.

6. Tertullien, Apologétique, 21,12-12, traduit par Jean-Pierre WALtzing et Albert Severyns, Paris, Les Belles Lettres (coll. « Universités de France »), 1961², p. 49-50.

7. Voir notamment Franz Joseph DöLGER, « Sonne und Sonnenstrahl als Gleichnis in der Logostheologie des christlichen Altertums », Antike und Christentum 1 (1929), p. 271290 ; É. BOUlarand, L'Hérésie d'Arius..., op. cit., t. 2, p. 313-321. 
Il n'est pas besoin de dire la récurrence et l'importance de la notion de lumière dans les écrits chrétiens des premiers siècles, mais il peut être intéressant, sur un corpus limité, de préciser davantage quel est l'usage et l'intérêt - du motif de la lumière en théologie. Parmi les Pères grecs qui en témoignent le mieux, on peut citer Clément d'Alexandrie, Origène $^{9}$, Grégoire de Nysse ou encore Syméon le Nouveau Théologien. Grégoire de Nazianze, quant à lui, ce Cappadocien devenu archevêque de Constantinople en 379 , a récemment été qualifié de « chantre de la Lumière », tant son œuvre en est remplie : comme le fait remarquer H. Alfeyev, la liturgie byzantine en la fête de saint Grégoire, le 25 janvier, le dit « réceptacle de la Lumière divine » et reconnaît en lui « le second Théologien [après saint Jean] et le maître initiant à la Lumière divine $\gg^{10}$. Or, comme nous proposons de le voir à partir de quelques textes précis, le ou les sens théologiques de la lumière chez le Théologien ne vont jamais sans un sens anthropologique.

\section{LE SENS THÉOLOGIQUE ET APOPHATIQUE DE LA LUMIÈRE}

Il semble y avoir chez Grégoire de Nazianze trois occurrences littérales de l'expression «lumière née de la lumière ». À chaque fois,

8. ARIUS, Lettre à Alexandre d'Alexandrie, traduite par Bernard MEUNIER dans Bernard SESBOÜÉ et Bernard MEUNIER, Dieu peut-il avoir un fils ? Le débat trinitaire du IV siècle, Paris, Éd. du Cerf (coll. « Textes en main »), 1993, p. 34 : « engendré, mais non pas [...] comme Hiéracas qui parle de lampe issue d'une lampe, ou comme un éclat lumineux qui se divise en deux ».

9. Voir Henri Crouzel, Origène et la «connaissance mystique », Paris, Desclée de Brouwer, 1961, p. 130-155 ; Francesco-Élie El KHOURY, Une ténèbre dans la lumière de Dieu. Le Père et son Fils d'après le Commentaire d'Origène sur saint Jean, Lyon, Profac, 2008, p. 56-59, 115-119.

10. Ode 1 et stichères des laudes, cités par Hilarion Alfeyev, Le Chantre de la Lumière. Introduction à la spiritualité de saint Grégoire de Nazianze, Paris, Éd. du Cerf (coll. «Théologies »), 2006, p. 384. Parmi les études les plus connues sur la lumière chez le Nazianzène, citons aussi Thomas SPIDLIK, Grégoire de Nazianze. Introduction à l'étude de sa doctrine spirituelle, Rome, Pontificum Institutum Studiorum Orientalium (coll. «Orientalia Christiana Analecta» 189), 1971, p. 15-47; Jean PlagnieuX, Saint Grégoire de Nazianze théologien, Paris, Éditions franciscaines, 1952, p. 276-332 (sur la question de l'apophatisme); Philippe Molac, Douleur et transfiguration. Une lecture $d u$ cheminement spirituel de saint Grégoire de Nazianze, Paris, Éd. du Cerf («Cogitatio fidei » 251), 2006, p. 273-280 (sur la « voie illuminative ») ; Claudio MORESCHINI, « Luce e purificazione nella dottrina di Gregorio Nazianzeno », Augustinianum 13 (1973), p. 535549. 
elle désigne le Fils dans la relation trinitaire, pour suggérer sa divinité. Ainsi dans le Discours 38, § $13^{11}$, exaltant

le Verbe de Dieu lui-même, celui qui est antérieur aux siècles, l'invisible, l'insaisissable, l'incorporel, celui qui est le Principe issu du Principe, la


de l'immortalité, l'empreinte du Modèle, le sceau immuable, l'image exacte, la définition et l'explication du Père.

La deuxième occurrence est dans le Poème II, $1,38^{12}$, v. 5-8, adressé au Christ :

Lumière du Père ( $\pi \alpha \tau \rho \circ \varphi \alpha \grave{\varepsilon} \varsigma)$, Verbe de la grande intelligence, au-delà des mots,

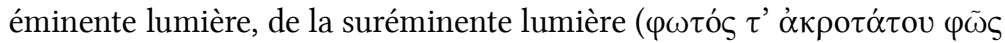
œ̋

image du Père immortel $(2$ Co 4, 4), sceau $(f n$ 6, 27) de l'être sans

principe,

lumière avec $(\sigma \nu \mu \varphi \alpha \grave{\varepsilon} \varsigma)$ le grand Esprit.

Le néologisme $\pi \alpha \tau \rho о \varphi \alpha \varepsilon ́ \varsigma$, créé par le poète, explicite en langage homérique la formule du symbole de foi, tandis qu'un autre néologisme, $\sigma \cup \mu \varphi \alpha \varepsilon \dot{\varepsilon}$, élargit son application à l'Esprit. Ce dernier trait est présent également dans la troisième occurrence, qui se trouve dans le Poème I, $1,32^{13}$, v. 3 - mais l'authenticité de ce poème, qui n'obéit pas à la métrique classique, est fortement contestée ${ }^{14}$ :

Nous te bénissons maintenant, mon Christ, Verbe de Dieu,

lumière de la lumière sans principe ( $\varphi \tilde{\omega} \varsigma \dot{\varepsilon} \kappa \varphi \omega \tau$ ò $\left.\alpha_{\alpha} v \dot{\alpha} \rho \chi 0 u\right)$

et dispensateur de l'Esprit,

troisième lumière unie

en une seule et même gloire!

11. GRÉGoIre De NAZIANZE, Discours 38-41, introduction, texte critique et notes par Claudio MoreschINI, traduction par Paul Gallay, Paris, Éd. du Cerf (coll. « Sources Chrétiennes » [=SC] 358), 1990, p. 132-133.

12. Jacques-Paul Migne, Sancti Patris nostri Gregorii Theologi vulgo Nazianzeni, archiepiscopi Constantinopolitani, opera quæ exstant omnia, t. III, Paris, Migne (coll. « Patrologie grecque » [= PG] 37), 1325-1326.

13. PG 37, 512.

14. Voir Hendrik Leonard DAVIDS, Die Gnomologieën van Sint Gregorius van Nazianze, Nijmegen-Utrecht, Dekker/Van de Vegt, 1940, p. 51-55; Hans Martin WeHrhan, «Dubia et spuria unter den Gedichten Gregors von Nazianz », Studia Patristica VII, Berlin, Akademie Verlag (coll. «Texte und Untersuchungen » 92), 1966, p. 337-348, spéc. p. 339-340. - Sauf exceptions dûment signalées, la traduction des Poèmes cités ici et numérotés d'après la classification de l'édition mauriste reproduite dans la Patrologie grecque, est personnelle. 
«L'Esprit, troisième lumière » : l'expression rejoint encore d'autres textes dans lesquels le motif christologique, qui est classique, s'enrichit nettement chez Grégoire d'un sens pneumatologique, au moment où la divinité du Saint-Esprit peine encore à s'imposer. Les emplois christologique et pneumatologique du thème de la lumière, chez le Nazianzène, sont bien moins importants, en réalité, que celui, trinitaire, qui leur donne sens. Ainsi dans le Discours 31, $\S 2^{15}$, où Grégoire, non sans audace, détourne en un sens triadologique un verset christologique :

Il était la vraie lumière qui éclaire tout homme venant dans le monde ( In 1,9), c'est le Père ; Il était la vraie lumière qui éclaire tout homme venant dans le monde, c'est le Fils; Il était la vraie lumière qui éclaire tout homme venant dans le monde, c'est l'autre Paraclet. Il était, et il était, et il était ; mais il était un. Il était lumière et lumière et lumière ; mais une seule lumière, un seul Dieu. C'est ce que David aperçut jadis, quand il disait: Dans ta lumière nous verrons la lumière (Ps 35,10$)$. Et nous, maintenant, nous avons vu et nous prêchons : de la lumière - le Père -, nous saisissons la lumière - le Fils -, dans la lumière - l'Esprit -, théologie brève et simple de la Trinité.

Le Poème II, 2, 4 ${ }^{16}$, v. 84-88, est moins problématique à cet égard :

Je ne verrai plus de loin les semblants de vérité altérés comme par un miroir ou à travers l'eau, mais je contemplerai avec des yeux purs la réalité elle-même, dont le commencement et la fin sont la Trinité, divinité à qui est rendu un seul culte ${ }^{17}$, unique lumière en trois rayonnements également divins.

Le thème est si récurrent et il est exprimé sur un ton souvent si personnel qu'on peut se demander si Grégoire, qui est au IV siècle celui qui a le plus approfondi la formulation de la Trinité, n'en a pas eu ce

15. Grégoire de Nazianze. Discours 27-31, introduction, texte critique, traduction et notes par Paul Gallay avec la collaboration de Maurice JouRJON, Paris, Éd. du Cerf (SC 250), 1978, p. 281 et la note. Voir aussi Discours 40, § 41, SC 358, p. 295 : «Lorsque je réunis les Trois dans la contemplation, je vois une seule splendeur, sans pouvoir distinguer ou mesurer la lumière qui est une »; Discours 31, § 14, p. 303 : «La divinité est sans division dans ceux qui sont distincts : de même, dans trois soleils se pénétrant mutuellement, unique est la fusion de la lumière » - Grégoire de Nazianze semble être à l'origine de cette image dont on peut suivre la trace dans la poésie liturgique notamment

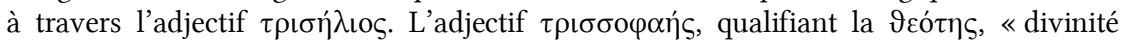
triplement lumineuse », dans les Poèmes I, 1, 4, v. 65 ; II, 1, 13, v. 214 ; II, 1, 99, v. 2 (PG 37, 421, 1244 et 1452), pourrait être également à l'origine des emplois ultérieurs.

16. PG $37,1512$.

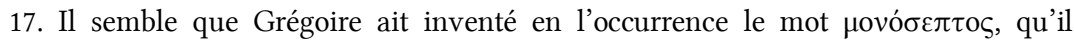
emploie aussi comme qualificatif de la Trinité dans le Poème II, 1, 16, v. 29. 
qu'on appellerait aujourd'hui une « expérience mystique ${ }^{18}$, au-delà de la simple heuristique intellectuelle et au plus profond de l'illumination, rituelle, du baptême. La fréquence du thème de la lumière associé à la Trinité est en tout cas un trait distinctif de l'œuvre de l'écrivain sacré, dont la théologie est très nettement « triadocentrée ».

De fait, à l'instar de Tertullien et de bien d'autres, la lumière est une image qui permet à Grégoire d'illustrer l'identité et l'égalité des trois personnes divines. L'image, cependant, n'est qu'une image. Dieu en tant que tel reste indicible. Il est donc nécessaire de se demander en quoi réside la lumière du Dieu chrétien et en quoi elle diffère de celle du Dieu gnostique ou manichéen, ou encore de tout autre déisme plus ou moins illuminé. Grégoire lui-même dénonce entre autres les cultes solaires et, en citant le premier récit de la création dans la Genèse, rappelle que la lumière est créée ${ }^{19}$. L'image du soleil, en outre, était utilisée par des hérétiques, comme Apollinaire, en un sens subordinationiste défavorable à la divinité du Saint-Esprit, et c'est ce que critique Grégoire dans la première Lettre théologique, $\S 67^{20}$ :

Si la Trinité se compose d'un grand, d'un plus grand et d'un très grand, comme d'une clarté, d'un rayon et d'un soleil, l'Esprit, le Fils et le Père ce qui est écrit clairement dans ses ouvrages -, c'est une échelle graduant la divinité, une échelle qui ne fait pas monter au ciel, mais qui jette à bas du ciel.

\section{Grégoire interroge son propre langage dans le Discours 31, $§ 32^{21}$ :}

J'ai imaginé - comme d'autres aussi - une source, un ruisseau et un fleuve, pour voir s'il y a une analogie entre la source et le Père, le ruisseau et le Fils, le fleuve et l'Esprit Saint. Ces choses ne sont pas, en effet, divisées par le temps, ni séparées l'une de l'autre au point de vue de

18. Lire par ex. le Discours 2, §7, dans Grégoire de Nazianze. Discours 1-3, introduction, texte critique, traduction et notes par Jean BERNARDI, Paris, Éd. du Cerf (SC 247), 1978, p. 97 : «Rien ne me paraissait aussi beau que de fermer la porte des sens, de sortir de la chair et du monde, de se ramasser sur soi-même, de n'avoir aucun contact avec les choses humaines en dehors d'une absolue nécessité, de s'entretenir avec soimême et avec Dieu, pour vivre au-dessus des réalités visibles, pour garder sur soi les reflets divins sans altération ni mélange d'aucune des empreintes de ce qui s'égare icibas, en étant et en devenant constamment vrai miroir immaculé de Dieu et des choses divines, en ajoutant lumière à lumière et en substituant la netteté à la confusion, en jouissant dès à présent par l'espérance des biens de la vie future, pour accompagner les anges dans leur ronde, en restant sur terre après avoir quitté la terre et avoie été élevé par l'esprit. Si l'un de vous est possédé de ce désir, il sait ce que je veux dire et il me pardonnera ce que j'ai alors éprouvé. »

19. Discours 28, § 13-15, SC 250, p. 127-133.

20. Grégoire de Nazianze. Lettres théologiques, introduction, texte critique, traduction et notes par Paul Gallay, Paris, Éd. du Cerf (SC 208), 1974, p. 65-67.

21. SC 250, p. 341-343. 
la continuité, et elles semblent se distinguer en quelque sorte par leurs trois propriétés. Mais j'ai craint d'abord de présenter par cette comparaison je ne sais quel écoulement de la divinité, qui exclurait la stabilité ; j'ai craint en second lieu d'introduire l'unicité (de personne), car la source, le ruisseau et le fleuve sont une seule chose qui prend des formes diverses. [...] Le mieux, c'est de laisser là les images et les ombres, qui sont trompeuses et très éloignées de la vérité, de m'attacher moimême à la pensée la plus conforme à la foi, de m'en tenir à un petit nombre de mots, de prendre pour guide l'Esprit, de garder jusqu'à la fin l'illumination que j'ai reçue (au baptême).

En d'autres termes, pour Grégoire l'usage des images - dont il ne se prive d'ailleurs pas - n'est pas sans limite et doit s'accompagner de nécessaires correctifs. Car l'abîme qui sépare Dieu de l'homme sera toujours infini, quelle que soit la valeur de l'analogie qui les rapproche. Le nom même de Dieu est inadéquat, malgré les étymologies, aussi classiques qu'erronées, que Grégoire invoque ${ }^{22}$ :

La divinité ne peut être désignée par aucun nom. [...] En effet, le mot «Dieu » (ó Э๕ós) a pour étymologie, d'après les gens subtils en cette

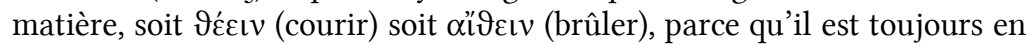
mouvement et parce qu'il consume nos dispositions perverses - c'est pourquoi il est appelé un feu dévorant $(D t 4,24)$; ce n'en est pas moins un mot d'un sens relatif et non absolu.

Grégoire le Théologien, le «Diseur de Dieu», avoue donc son impuissance. Il est, du reste, l'auteur reconnu ou présumé de textes parmi les plus connus en matière de théologie négative ou apophatique ${ }^{23}$. C'est que, loin de se réduire à une idée vide, ou à un concept par défaut, et plus loin encore d'être un simple motif pour figures rhétoriques ou poétiques (ce n'est pas le lieu ici d'énumérer les antithèses, oxymores, répétitions d'adjectifs commençant par l'alpha privatif et autres accumulations de négations), l'apophatisme a chez Grégoire un statut positif. Citons à ce propos le Discours $38, \S 7^{24}$ :

À mon sens, la divinité veut, en tant qu'elle est saisissable, attirer à elle car ce qui est parfaitement insaisissable n'est pas objet d'espérance et on ne cherche pas à l'atteindre-; mais aussi, en tant qu'elle est insaisissable, elle veut provoquer l'admiration; étant admirée, elle est plus désirée; étant désirée, elle purifie; en purifiant, elle rend aussi semblable à Dieu ; avec ceux qui en sont arrivés là, Dieu entretient des

22. Discours 30, § 17-18, SC 250, p. 263-265.

23. Parmi eux le fameux hymne à Dieu (Poème I, 1, 29), dont l'authenticité est si débattue : « Ô toi, l'Au-delà de tout, est-il permis de te chanter autrement ? Comment la parole te célébrera-t-elle, toi qu'aucune parole ne saurait dire? »

24. SC 358, p. 117. 
relations d'intimité ; et - je parle ici avec une certaine audace - Dieu s'unit à des dieux, il en est connu, et peut-être autant qu'il connaît déjà ceux qui sont connus de lui. La divinité est donc sans limites et difficile à contempler. Ce qui est entièrement saisissable en Dieu, c'est seulement qu'il est sans limites.

Cette dernière affirmation est l'expression la plus claire de l'apophatisme, qui, comme on le voit, est l'objet de la volonté divine ellemême. Le rôle de l'admiration ou de l'étonnement ( $\tau$ ò $\vartheta \alpha u \mu \alpha ́ \alpha \zeta \varepsilon ı v$ ) est ici à souligner en tant qu'étape nécessaire, non seulement vers la philosophie, comme chez Platon, mais vers la déification. Si l'on songe au célèbre passage du Théétète, $155 \mathrm{~d}$, où l'étonnement est reconnu comme la « vraie marque du philosophe », et où Iris, messagère des dieux dont l'arc-en-ciel est la manifestation, est évoquée comme fille de Thaumas (« l'étonnement » personnifié), le Discours 38 résonne de façon incidente avec le Poème I, 1, 7, l'un des plus beaux textes de Grégoire sur la lumière divine éclairant l'intelligence humaine ${ }^{25}$ :

On voit parfois à travers l'air humide et serein un rayon de soleil rencontrer des nuages en formant des cercles qui se repoussent mutuellement ;

un arc-en-ciel multicolore se déroule et tout autour l'éther s'illumine en d'innombrables cercles, aussitôt détruits.

C'est une image des lumières célestes; la Lumière suprême illumine sans cesse de ses rayons les intelligences inférieures. La source des rayons, c'est cette Lumière dont le nom est ineffable, et qu'on ne peut saisir, car elle échappe à l'esprit qui veut l'approcher, si rapide soit-il,

et elle ne cesse de se dérober à toutes les intelligences, afin que nos désirs nous fassent tendre à une hauteur toujours nouvelle.

Là encore, les limites humaines sont présentées comme un effet de la Providence. Le Discours $32, \S 15$, entre encore plus profondément dans la question, faisant de l'apophatisme le signe d'une pédagogie divine ${ }^{26}$ :

Dieu est lumière $(\exists n 1,5)$, et la plus haute lumière ; un faible écoulement, un faible rayonnement venant jusqu'ici-bas, c'est toute notre lumière, encore qu'elle nous paraisse très brillante ; mais, vois-tu, Dieu foule notre obscurité, et il a placé les ténèbres comme sa retraite $(P s 17,12)$ entre lui et nous; comme jadis Moïse plaça aussi le voile entre lui-même et l'endurcissement d'Israël (voir Ex 34,33): c'est pour que notre nature

25. Poème I, 1, 7, v. 1-11, PG 37, 438-439, dans Grégoire de Nazianze. Poèmes et lettres choisis et traduits avec introduction et notes par Paul GALLAY, Lyon-Paris, Emmanuel Vitte (coll. «Les grands écrivains chrétiens »), p. 131-132.

26. Grégoire de Nazianze. Discours 1-3, introduction, texte critique, traduction et notes par Paul Gallay, Paris, Éd. du Cerf (SC 318), 1985, p. 117-119. 
enténébrée ne voie pas facilement la beauté cachée et que bien peu méritent de voir; c'est aussi pour éviter que, si nous l'atteignons facilement, nous ne la perdions facilement aussi, à cause de l'aisance qu'il y aurait à l'acquérir; il faut que notre lumière prenne contact avec la Lumière - cette dernière l'attirant sans cesse vers les hauteurs par le désir -, il faut que notre esprit purifié s'approche de la pureté absolue et qu'une partie de celle-ci lui apparaisse maintenant, et le reste plus tard, en récompense de la vertu, de l'élan d'ici-bas vers cette pureté absolue, ou plutôt de l'assimilation à elle.

Autrement dit, l'obscurité de Dieu n'est pas un résultat direct de la bassesse humaine (incapable d'ailleurs d'affecter la divinité), mais bien un moyen pédagogique pour l'élever et accomplir la promesse divine.

L'obscurité est donc comprise comme un bienfait; c'est ce qu'il dit explicitement dans le Discours 28, §12: « Ne pas avoir le bienfait à

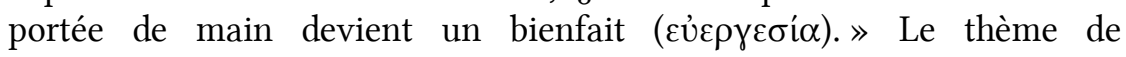
l'assimilation, qui est celui de l'image en $G n 1,26$, nous renvoie ici aux récits de la création, et même si l'arbre de la connaissance $(G n 2,9)$ n'est pas mentionné, la perspective prend bien une ampleur protologique et eschatologique. L'arbre interdit aux origines est promis à l'homme ; l'interdit ou l'obscurité ne vise pas à en écarter l'homme absolument, mais bien à lui permettre de l'atteindre en dernier ressort, selon ses possibilités et son désir. L'homme et la femme ne sont pas appelés à être comme des dieux $(G n 3,5)$, mais à devenir réellement des dieux en Dieu et par Dieu.

Le sens profond de l'apophatisme commence ici à se laisser deviner : car enfin, n'est-il pas étrange de voir l'apophatisme de Grégoire se fonder, non sur une négation, mais sur une affirmation: «Dieu est lumière » ? De même que les ténèbres font paraître plus brillante encore la lumière, la reconnaissance des limites humaines est pour le Théologien la condition nécessaire à l'authentique confession de l'infinité divine.

De fait, il est significatif de remarquer que les théologiens qui, avec Grégoire, ont fait émerger et se confirmer le langage dogmatique, ont bâti leurs dogmes sur ce qui pour eux est au-delà de tout dogme; et ce $\mathrm{IV}^{\mathrm{e}}$ siècle qui a défini sa foi de façon si élaborée a donné pour socle à toute théologie future un insaisissable Infini.

Il n'y a pas, en somme, deux langages contradictoires chez Grégoire, l'apophatique s'opposant au cataphatique ; ils visent tous deux un unique objet, dont seul l'excès dépasse les limites humaines. Terme privilégié dans l'expression de la divinité, la lumière permet bien de faire le lien entre une formulation positive de Dieu («Dieu est lumière ») et une vision en «négatif » (Dieu éblouit) : les théologies dites apophatique et cataphatique sont une seule et même théologie. À ce titre il est éclairant de comparer Grégoire de Nazianze à Grégoire de Nysse, qui a développé 
davantage encore la théologie de la nuée, celle qui couvre les Hébreux menés par Moïse. Thomas Spidlik a justement noté qu'à la différence du Nazianzène, pour qui la «nuée » est un obstacle, le Nyssène en fait une forme paradoxale et suprême de révélation : «Le regard de Moïse a pénétré dans la ténèbre ( $\gamma$ vó $\varphi \circ \varsigma)$ et a contemplé en elle l'Invisible ${ }^{27}$. Pour le reste, Claudio Moreschini constate une plus grande rationalité, une plus ample systématisation du thème chez l'auteur de la Vie de Moïse et souligne «la différence entre la personnalité bien plus enthousiaste et ardente du Nazianzène et celle, plus froide et rationnelle, $\mathrm{du}$ Nyssène $»^{28}$. Hilarion Alfeyev, quant à lui, fait « remarquer que, tandis que l'idée de la Lumière divine est devenue centrale dans la tradition orientale à partir de Grégoire de Nazianze, celle des ténèbres de Dieu, caractéristique de Grégoire de Nysse, est restée à la périphérie de la théologie byzantine. De façon générale, la théologie orientale a plus suivi Grégoire de Nazianze que Grégoire de Nysse aussi bien dans les questions doctrinales que mystiques. ${ }^{29}$

Quoi qu'il en soit, apophatisme et théologie positive sont bien articulés chez Grégoire de Nazianze autour de la médiation du Christ. L'emploi du motif de la lumière en un sens christologique est à cet égard très significatif chez Grégoire: témoignant d'une christologie descendante, il a souvent une visée sotériologique, dans la perspective de l'économie du salut. Dieu, écrit Grégoire dans le Discours 37, $\S 4^{30}$, peut être saisi et même dit parce qu'il l'accepte, parce que dans l'Incarnation il a fait siens la nature humaine et son langage, même si celui-ci est audessous de lui : «Tu es appelé Verbe, et tu es au-dessus du verbe; tu es au-dessus de la lumière, et tu es nommé lumière ».

Le renversement est donc complet. L'inaccessible se fait accessible, par un mouvement de double assimilation : Dieu s'est rendu semblable à l'homme pour que l'homme soit rendu semblable à lui ; et de même que l'objet que la lumière éclaire devient lui-même lumineux, c'est en se tournant vers Dieu que l'homme le connaîtra et sera assimilé à lui. Le Discours $38, \S 13^{31}$, va plus loin encore en dévoilant en quoi consiste la lumière de ce Dieu non seulement créateur, mais incarné et crucifié :

27. In Psalmum septimum, PG 44, 457A, cité par Th. SPIDLIK, Grégoire de Nazianze..., op. cit., p. 44-45.

28. C. MORESCHINI, « Luce e purificazione... », art. cit., p. 549.

29. H. Alfeyev, Le Chantre de la Lumière..., op. cit., p. 292.

30. SC 318, p. 279.

31. SC 358, p. 135. Ce passage est cité par MAXIME LE Confesseur, Questions à Thalassios 54, traduites par Jean-Claude LARCHET, Paris, Éd. du Cerf (SC 554), 2012, p. 210, lignes 264-267 (où la citation n'est pas identifiée); Maxime prolonge le raisonnement jusqu'à la divinisation et à l'« ineffable recréation finale », p. 213. 
Il instaure avec nous une seconde communauté, bien plus extraordinaire que la première : il nous avait donné part à ce qui est supérieur (son image), maintenant il participe à ce qui est inférieur (la chair); cet état est plus digne de Dieu que le premier, cet état, pour ceux qui savent comprendre, est plus élevé.

Plus que par la voie ascendante - celle de la ressemblance à Dieu visée par le récit de la Genèse -, la lumière divine apparaît davantage ici par la voie descendante, c'est-à-dire dans l'Incarnation, dans la faiblesse humaine assumée par le Verbe divin : au contraire de la gnose, la lumière chrétienne est à chercher dans l'obscurité du monde.

\section{LE SENS ANTHROPOLOGIQUE DE LA LUMIÈRE}

Il y a pour le Théologien, on le voit, un double lien entre l'homme et Dieu : celui de la création «à l'image et à la ressemblance », et celui de l'Incarnation de Dieu assumant la nature humaine. Dès lors, en régime chrétien, toute théologie devient aussi une anthropologie, l'être humain n'ayant, pour les Pères, qu'à regarder vers Dieu pour savoir quel homme il est ou est censé être. La création à l'image n'est d'ailleurs qu'une préfiguration de l'Incarnation, qui n'est autre que la manifestation de l'image parfaite de Dieu, image dont l'homme est à son tour l'image ; or, même si c'est bien l'Incarnation qui, en théologie chrétienne, fonde la création à l'image, bien des auteurs patristiques, tels des philosophes, expliquent la ressemblance humaine à Dieu par la seule dépendance ontologique et par une commune nature spirituelle ou intelligible.

Pour le Nazianzène, la lumière est bien en l'homme l'un des motifs de la ressemblance à Dieu ${ }^{32}$. Dans le Discours 40, sur le baptême, qui est une sorte de traité de la lumière, ou plutôt des lumières ${ }^{33}$, il brosse un tableau grandiose de la totalité des êtres selon un schéma ontologique descendant et hiérarchisé. La concaténation et l'énumération semblent

32. Voir aussi Henri Crouzel, Théologie de l'Image de Dieu chez Origène, Paris, Aubier (coll. « Théologie » 34), 1956, notamment p. 87-90, 161, 168.

33. Discours 40, §5, SC 358, p. 205-207. Voir le «mémento» tiré de ce passage et intitulé De XIV luminibus iuxta Nazianzeni recensionem (Clavis Patrum Graecorum 3094), édité par Jean-Baptiste PITRA, Spicilegium Solesmense complectens Sanctorum patrum scriptorumque ecclesiasticorum anecdota hactenus opera, t. III, Paris, Firmin

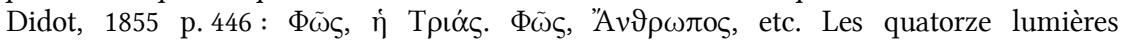
énumérées, un peu différentes de celles du Discours 40 puisque l'ange n'y figure pas, sont : la Trinité, l'homme, la lumière $(G n 1,3)$, le premier commandement $(G n 2,16)$, la Loi écrite, le buisson ardent $(E x 3,2)$, la colonne de feu $(E x$ 13, 21), l'enlèvement d'Élie sur un char de feu $(2 R 2,11)$, l'étoile des bergers $(L c 2,9)$, celle des mages $(M t 2,9)$, la Transfiguration $(M t 17,2)$, l'apparition à Paul $(A c 9,3)$, la béatitude de l'au-delà $(S g 3,7)$, le baptême. 
mimer ici l'universelle diffusion de la lumière divine, inondant, structurant les êtres dans leur constitution même :

Dieu est la lumière suprême, inaccessible et inexprimable ; elle n'est ni comprise par l'esprit, ni exprimée par la parole; et elle illumine toute nature douée de raison. Cette lumière est dans le monde intelligible ce que le soleil est dans le monde sensible; dans la mesure où nous sommes purifiés, elle nous apparaît ; dans la mesure où elle nous apparaît, elle est aimée de nous; et dans la mesure où nous l'aimons, en retour nous la connaissons ; elle se contemple et se comprend elle-même et se répand peu à peu dans ce qui est extérieur à elle. Je parle de la lumière qui est contemplée dans le Père, le Fils et le Saint-Esprit; leur richesse, c'est l'identité de nature et le jaillissement unique de leur splendeur.

La deuxième lumière, c'est l'ange qui est une sorte d'écoulement de la première Lumière ou de participation à elle; parce qu'il a une propension vers elle, et qu'elle le soutient, il tient d'elle son illumination. [...]

La troisième lumière, c'est l'homme, et cela est manifeste même aux gens du dehors : en effet, ils donnent à l'homme le nom de phôs (lumière) à

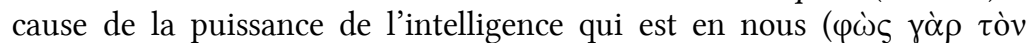

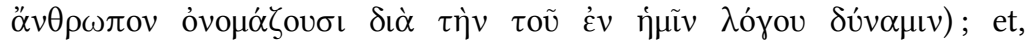
inversement, ce nom est donné à ceux d'entre nous-mêmes qui sont plus semblables à Dieu et qui s'approchent plus de Dieu.

Et Grégoire d'énumérer ensuite la lumière dans la création visible, celle du «premier précepte donné au premier homme», celle de la loi écrite, etc. Le texte résonne de plus d'un écho platonicien: le soleil intelligible (République VI, 508c), la participation ( $\mu \varepsilon \tau o v \sigma i ́ \alpha$ ), l'assimilation à Dieu par le logos ou l'intellect dont la nature est plus proche du divin ${ }^{34}$, l'assimilation visuelle aussi, qui est au cœur de la théorie platonicienne de la vision ${ }^{35}$.

Cette page mériterait à elle seule un très long commentaire; nous nous concentrerons sur la mention de l'emploi que les poètes antiques faisaient du mot masculin $\varphi \omega \varsigma^{36}$ pour désigner l'homme. La remarque a un précédent en patristique grecque, chez Clément d'Alexandrie ${ }^{37}$ :

Celui qui vient à peine d'être régénéré, celui-là, comme son nom l'indique, il a été illuminé et aussitôt il a été libéré de l'obscurité et, de ce fait même, il a reçu la lumière. Comme ceux qui, après avoir chassé le 27.

34. Sur ce thème, voir notamment Th. SPIDLIK, Grégoire de Nazianze..., op. cit., p. 21-

35. «Le semblable rencontre le semblable », Timée $45 \mathrm{c}$.

36. Le mot a un accent aigu ou grave, et non circonflexe comme dans le mot neutre $\varphi \tilde{\omega} \varsigma$, « lumière », mais, nous allons le voir, les copistes n'y attachaient pas d'importance.

37. Le Pédagogue, I, vI, 27,3-28,2, traduit par Marguerite HarL, Paris, Éd. du Cerf (SC 70), 1960, p. 163. 
sommeil, se trouvent du fait même réveillés, ou plutôt comme celui qui cherche à enlever la buée de ses yeux n'obtient pas de l'extérieur la lumière dont il était privé mais enlève seulement ce qui obstruait ses yeux et rend la pupille libre, ainsi, lorsque nous sommes baptisés, étant débarrassés des fautes qui, à la manière d'un nuage, faisaient obstacle à l'Esprit divin, nous rendons libre, sans voile et lumineux, cet œil de l'esprit qui seul nous fait contempler le divin, car le Saint-Esprit venant du ciel s'écoule en nous : c'est un onguent de clarté éternelle capable de faire voir la lumière éternelle, car le semblable est ami du semblable. Et ce qui est saint est ami de cela d'où vient la sainteté, appelée, au sens propre, la « lumière »: Car vous avez été un jour ténèbres, mais vous êtes maintenant lumière dans le Seigneur $(E p 5,8)$. Et c'est pourquoi, je pense,

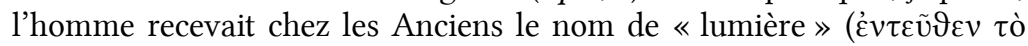

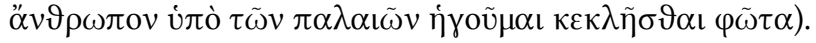

Là encore, le contexte est baptismal ; là encore, l'arrière-plan est platonicien ; là toujours, la lumière est avant tout intelligible : il semblait aller de soi que l'identification de l'homme à la lumière avait une explication « logique », au double sens, noétique et linguistique, du mot grec $\lambda$ óyos. Si, pour des spécialistes modernes comme P. Chantraine, l'étymologie du mot $\varphi \omega ́ s$ est « obscure ${ }^{38}$ - de même que celle du mot « dieu », $\theta \varepsilon$ ó $\varsigma$, reste « inconnue ${ }^{39}$-, pour les Anciens et les Byzantins, en revanche, l'étymologie était pleine de sens : ainsi pour le PseudoZonaras vers le XIII ${ }^{\mathrm{e}}$ siècle ${ }^{40}$, si $\varphi \omega \omega_{\zeta}$ veut dire «homme », c'est parce qu'il est « le seul à faire briller les choses de la pensée par la parole $(\tau \tilde{\omega}$ $\lambda \operatorname{ó}_{(}(2)$, ou parce qu'il dérive du mot "dire", "parler" : car l'homme est un

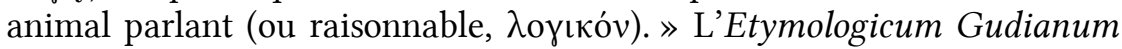
reprend cette étymologie en citant le passage du Discours 40, où l'occurrence du mot $\lambda$ óyou, traduite plus haut par «intelligence », penche plutôt vers le sens de $\ll$ parole $»^{41}$; ce contexte linguistique ne

38. Pierre ChAntraine, Dictionnaire étymologique de la langue grecque, Paris, Klincksieck, 1980, p. 1238 : « $\Phi \omega ́ \varsigma, \varphi \omega \tau$, : m. "homme, héros, mortel” (Hom., trag., poètes) ; jamais employé au f. [...] Ét. : Obscure. Si la flexion en dentale est secondaire, identité formelle entre le nom. grec et skr. bhas- n. "lumière, éclat, majesté", Mayrhofer, Et. Wb. 2, 499 ; mais du point de vue sémantique le rapprochement serait malaisé. »

39. Ibid., p. 430.

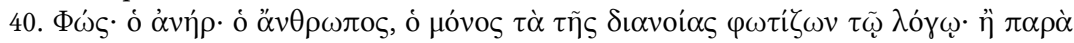

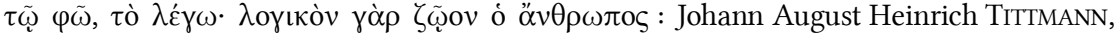
Iohannis Zonarae lexicon ex tribus codicibus manuscriptis, vol. 1, Leipzig, Crusius, 1808 (réimpr. Amsterdam, Hakkert, 1967), p. 1835. La source pourrait être APOLLONIUS LE SopHISTE ( $\left(\mathrm{I}^{\mathrm{er}}-\mathrm{II}^{\mathrm{e}} \mathrm{s}.\right)$, Lexicon Homericum, éd. Immanuel Bekker, Apollonii Sophistae lexicon Homericum, Berlin, Reimer, 1833 (réimpr. Hildesheim, Olms, 1967), p. 166 : $\varphi \tilde{\omega} \tau \varepsilon \varsigma$ oi

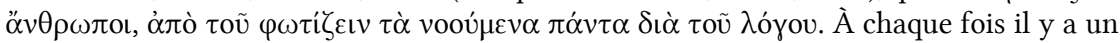
jeu sur le double aspect noétique et linguistique du $\lambda$ ó yos.

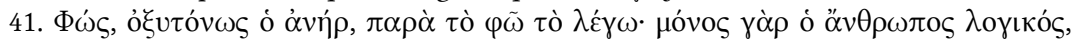

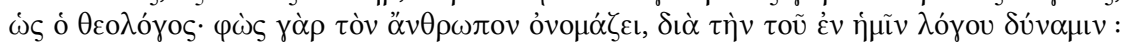


serait d'ailleurs pas incompatible avec un sens christologique si l'on voulait traduire par « Verbe ».

Il est assez curieux de constater un emploi très récurrent, presque constant, de $\varphi \omega ́$ chez Homère et jusque dans la poésie tardive ${ }^{42}$, où il est qualifié par l'adjectif i $\sigma o ́ \theta \varepsilon \varepsilon^{43}$, «égal à un dieu»ou, chez les auteurs chrétiens ${ }^{44}$, «égal à Dieu ». Par contrepoint, la condition de mortel marque bien le sens du mot $\varphi \omega ́ s$, mais la relation, l'équivalence au divin conviendrait parfaitement au propos du poète cappadocien.

Lui-même emploie parfois le mot $\varphi \omega ́ s$, notamment au v. $631 \mathrm{du}$ Poème II, $1,1^{45}$, où il résonne très nettement avec le mot lumière au vers précédent :

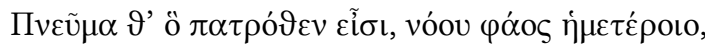

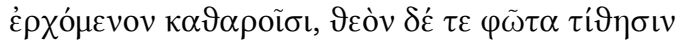

630 Et toi, Esprit qui viens du Père, lumière de notre intelligence,

631 toi qui descends sur les purs et qui fais de l'homme un dieu...

Or, cinq vers plus haut, v. 625 , on trouve une expression très audacieuse : «Fils immortel, grande lumière ${ }^{46}$ d'une lumière

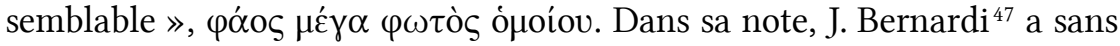
doute raison de justifier sa traduction - et l'éventuelle audace du Théologien-, en arguant du fait que le mot «semblable» ici ne s'applique pas à la substance du Père, mais à la lumière, «qui n'est qu'un attribut de la substance ». D'après le parallèle du v. 630, il est peut-être plus simple de comprendre $\varphi \omega \tau$ ò ónoíov comme désignant non pas le Père, mais l'homme créé à la ressemblance de Dieu. Puisque la forme est identique au génitif pour les mots $\varphi \tilde{\omega} \varsigma$ et $\varphi \omega ́ s$ et que, de

Friedrich Wilhelm STURZ, Etymologicum Graecae linguae Gudianum et alia grammaticorum scripta e codicibus manuscriptis nunc primum edita, Leipzig, Weigel, 1818 (réimpr. Hildesheim, Olms, 1973), p. 560.

42. HoMÈRe, Iliade II, 565 ; III, 310 ; IV, 212 ; VII, 136 ; IX, 211 ; XI, 472. 644 ; XV, 559 ; XVI, 632 ; XXIII, 569.677 ; Odyssée I, 324 ; XX, 124 ; EschYle, Perses, 80 ; Oracles sibyllins, V, 139 ; EudocIE, Centons homériques I, 447. 1606. 2286 ; II, 1471 ; III, 432 ; IV, 381.549 ; V, 395. 571.

43. On trouve aussi, de façon comparable, $\alpha \dot{v} \tau i \theta \varepsilon$ os $\varphi \omega ́ \varsigma$, Oracles sibyllins, XI, 276, ou

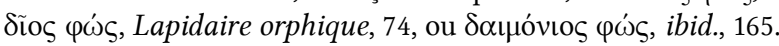

44. Voir par exemple GRÉGOIRE DE NySSE, Sur les morts, éd. Gunter HeIL, Gregorii Nysseni Sermones, Pars I, Leyde, Brill (coll. « Gregorii Nysseni Opera » IX), 1967, p. 54,

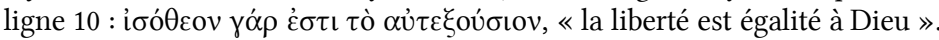

45. Saint Grégoire de Nazianze. Euvres poétiques. Tome 1, $1^{\mathrm{re}}$ partie. Poèmes personnels II, 1, 1-11, texte établi par André TUILIER et Guillaume BADY, traduit et annoté par Jean BERNARDI, Paris, Les Belles Lettres (coll. « Universités de France »), 2004, p. 43.

46. Comme on l'a vu, le Fils est parfois désigné comme «grande lumière » : ainsi dans le Discours 40, § 37, ou les Poèmes I, 1, 18, v. 41, et II, 1, 34, v. 110.

47. Ibid., n. 165. 
surcroit, l'usage semblait confondre de toute façon les deux mots, le poète aurait ménagé ici une ambiguité volontaire.

Un tel jeu délibéré trouve en tout cas un équivalent au $\mathrm{VI}^{\mathrm{e}}$ siècle au chapitre 3 des Questions et réponses du Pseudo-Césaire ${ }^{48}$, dont le Nazianzène est l'une des sources :

Lumière, le Père, lumière, le Fils, lumière, l'Esprit divin, mais les trois sont une seule lumière supratemporelle, préexistante, sans principe,

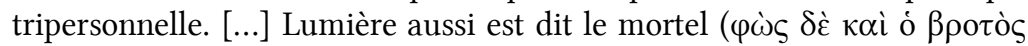
$\pi \rho о \sigma \alpha \gamma о \rho \varepsilon v ́ \varepsilon \tau \alpha \iota)$, lumière aussi le soleil ( $\varphi \tilde{\omega} \varsigma \kappa \alpha \grave{i}$ ò $\dddot{\eta} \lambda ı \varsigma)$ et la lune et le chœur chamarré des astres, mais de ces très nombreuses et diverses lumières, le démiurge qui les a créées à profusion de formes et de natures est la lumière véritable et immatérielle, le Dieu et Père de tous avec son Fils unique et l'Esprit divin.

Il serait peut-être plus simple, en l'occurrence, d'écrire $\varphi \tilde{\omega} \varsigma \delta \grave{\varepsilon}$.

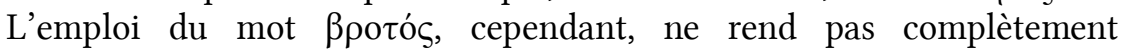
improbable ici l'usage du mot poétique $\varphi \omega ́ s$. De façon significative, dans ce cas comme au v. 625 du Poème II, 1, 1, la traduction par «homme » rend le texte difficilement intelligible ${ }^{49}$.

Un autre passage, au v. 7 du Poème I, 1, 11 $1^{50}$, «Sur l'incarnation du Christ, contre Apollinaire», pose un problème comparable pour l'établissement du texte :

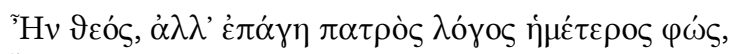

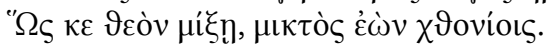

7 Il était Dieu $(\mathcal{F} n 1,1)$, mais le Verbe du Père s'est fait notre homme,

8 afin de mêler Dieu en étant mêlé aux êtres terrestres.

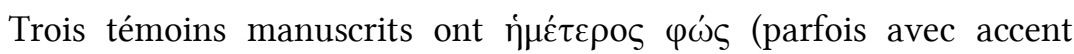
circonflexe), leçon retenue par Armand Benjamin Caillau et reproduite dans la Patrologie de Jacques-Paul Migne, six ont la leçon facilitante $\dot{\eta} \mu \varepsilon \dot{\tau} \tau \varepsilon \rho 0 v \quad \varphi \tilde{\omega} \varsigma$, «notre lumière », qui n'est pourtant pas sans

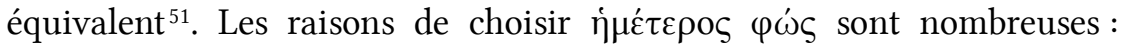
indépendamment du contexte, $\dot{\eta} \mu \varepsilon \dot{\tau} \varepsilon \rho \circ \varsigma$ $\varphi \omega ́ \varsigma$ est une lectio difficilior et, en tant qu'attribut du sujet du verbe غ̇ंáxy, elle offre un sens meilleur;

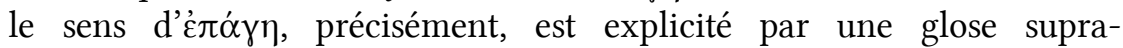
linéaire - en petites onciales - de l'antique Clarkianus 12, f. 144v,

48. Rudolf RiEdinger, Pseudo-Kaisarios. Die Erotapokriseis, Berlin, Akademie Verlag (coll. « Die griechischen christlichen Schriftsteller der ersten Jahrhunderte »), 1989, p. 14.

49. De même, dans le De XIV luminibus iuxta Nazianzeni recensionem cité plus haut et inspiré du Discours 40, le jeu de mots est indéniable.

50. PG 37, 471.

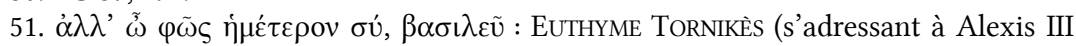
Comnène), Discours 1, 19, ligne 10, éd. Jean DARROUZÈs, «Les Discours d'Euthyme Tornikès (1200-1205) », Revue des Études Byzantines 26 (1968), p. 49-121, spéc. p. 71. 


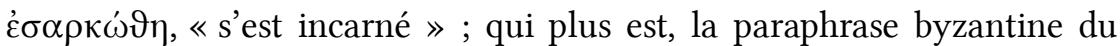
Marcianus gr. 82, du XIII siècle, f. 128, rend $\varphi \tilde{\omega} \varsigma$ (ainsi accentué) par

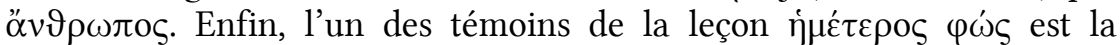
Doctrina patrum, attestée ici par trois manuscrits et citant le poème comme douzième pièce du ch. 3 intitulé : «Que le nom du Christ désigne les deux natures, et que le Christ est Dieu et la Vierge Mère de Dieu, contre Nestorius l'athée. $\gg^{52}$ Dans un tel contexte, il paraît clair que dès le VII siècle, si ce n'est avant, le mot $\varphi \omega ́ s$ de ce vers ne pouvait être compris que dans le sens d'« homme ».

À la faveur du jeu de mots la lumière est mise en relief comme constitutive de l'humanité. Par une révélation pour ainsi dire «photographique », l'homme trouve sa propre identité en Dieu, ainsi que le chemin de sa divinisation. L'expression «lumière (née) de la lumière », marquant un engendrement éternel et proprement théologique, a dès lors un enjeu « économique » ou sotériologique : les êtres humains peuvent à leur tour revendiquer la filiation de la lumière. Grégoire le dit explicitement en se référant à Paul dans le Discours 39, $\S 2^{53}$ :

Ainsi pourrons-nous rejeter les ténèbres, accéder à la lumière et devenir lumière parfaite, fils de la lumière $(E p 5,8)$ parfaite.

L'homme ne reçoit donc pas seulement la lumière, il est lumière. Déjà cité malgré son inauthenticité probable, le Poème I, $1,32^{54}$, v. 13-18, est très clair à ce sujet :

Tu as illuminé la pensée de l'homme

par la raison et la sagesse,

en plaçant ici-bas l'image

de la splendeur d'en haut,

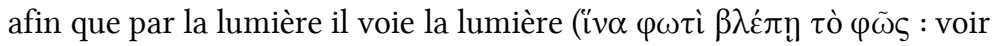

Ps 35,10$)$

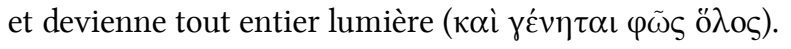

La portée morale, pratique est dès lors immédiate, comme dans le Discours 40, $\S 37^{55}$ :

Devenons lumière, comme les disciples reçurent ce nom quand la grande Lumière leur disait : Vous êtes la lumière du monde $(M t 5,14)$. Devenons

52. Franz DieKAMP, Doctrina Patrum de Incarnatione Verbi. Ein griechisches Florilegium aus der Wende des siebenten und achten Jahrhunderts, Münster, Aschendorff, 1907, p. 28.

53. SC 358, p. 153.

54. PG 37, 512

55. SC 358, p. 285. 
des flambeaux dans le monde, tenant la parole de vie (Ph 2,15-16), c'està-dire une puissance de vie pour les autres.

Or pour Grégoire en particulier, la ville qui ne peut être cachée, ou la lampe à ne pas laisser sous le boisseau si l'on suit $M t 5,14-15$, c'est la Trinité elle-même, qu'il a, de fait, contribué à faire briller dans les esprits de son époque et pour longtemps. Le Poème II, 1, 37 $7^{56}$, v. 3-4, le dit bien :

La Trinité le sait, elle que j’ai annoncé à la Ville en rallumant l'étincelle jusque-là enfouie.

Le Théologien fait ici référence à Constantinople et notamment au concile de $381^{57}$, lorsqu'il eut à défendre face à ses propres confrères l'égale divinité du Père, du Fils et de l'Esprit. C'est de là surtout que lui viennent les titres déjà évoqués que la liturgie byzantine lui réserve: Grégoire est dit non seulement « réceptacle », mais aussi « maître de la Lumière divine ». Sa personnalité suit à sa façon la trajectoire des rayons divins. Car le schéma de l'exitus et du reditus, ou de la descente et de la remontée de la lumière divine dans l'humanité, s'accomplit sur tous les plans : non seulement intelligible ou ontologique, mais aussi éthique et sotériologique.

Il est possible que l'image nicéenne de la «lumière [issue] de la lumière» ait marqué, comme l'écrit J. Moingt, un «tournant de civilisation ». Il est probable que la connotation intellectuelle qu'avait cette image pour des Grecs a infléchi la vision de Dieu en un sens noétique. Il est sûr, en tout cas, que, des religions solaires au judaïsme où, même si la lumière ne désigne pas directement Dieu, elle est associée au divin -, du platonisme au christianisme, des Lumières aux illuminés de tout bord, la lumière est un langage presque universel, un bien commun à de nombreuses civilisations, qu'il y ait ou non des «tournants ».

Triadologie, christologie, anthropologie : le champ sémantique de la lumière est tellement large, ne serait-ce qu'en christianisme, il semble si commun à toutes les religions et il a un tel pouvoir d'assimilation... Ce

56. PG 37, 1324.

57. Voir Poème II, 1, 93, v. 9, PG 37, 1448 : «J'ai amené la Trinité, Seigneur, à la plus jeune Rome»; Poème II, 1, 15, v. 9, «Sur lui-même, après son retour de Constantinople », PG 37, $1251:$ «La commune splendeur de la grande Trinité, que j'étais venu révéler jusqu'à de nobles étrangers »; Poème II, 1, 96, v. 2, PG 37, 1450 : « Grégoire, serviteur de la sainte Trinité »; Poème II, 1, 12, v. 117, PG 37, 1174 : «J'ai fait briller la Trinité à ceux qui jusque-là étaient dans les ténèbres »; Poème II, 1, 14, v. 49-50, PG 37, 1248-1249 : «Puissé-je briller comme une lampe sur le lampadaire, puissé-je illuminer le monde entier! » 
langage pourrait-il servir à dire n'importe quoi ? Le risque est limité chez Grégoire de Nazianze, qu'il s'agisse de parler du paradoxe trinitaire, de trouver dans l'obscure humanité la lumière divine ou - plus délicat encore -, de passer de la première à la seconde. Le symbole de Nicée en témoigne : la lumière n'est pas seulement une expression positive et même privilégiée de l'apophatisme, mais par rapport aux vocables du « Père » et du «Fils », ou à ceux du « Verbe » ou de la «Parole», elle constitue une alternative authentique et féconde pour dire quelque chose du Dieu des chrétiens.

RÉsumé. - La lumière, image de Dieu et nom de l'homme chez Grégoire de Nazianze. Par Guillaume BADY.

Dans la deuxième moitié du IV siècle, Grégoire de Nazianze, plus tard surnommé «le Théologien », est l'un des Pères grecs chez qui le thème de la lumière est le plus présent. Terme privilégié dans l'expression de la divinité, la lumière permet de faire le lien entre une formulation positive de Dieu («Dieu est lumière ») et une vision en «négatif » (Dieu éblouit): les théologies dites apophatique et cataphatique sont une seule et même théologie. Du côté anthropologique, l'incarnation du Fils, «lumière née de la lumière », donne un sens nouveau à l'un des mots qui, en grec, désignent l'être humain: le mot phôs. Par une révélation pour ainsi dire «photographique», l'homme trouve dès lors sa propre identité en Dieu, ainsi que le chemin de sa divinisation.

MOTS-CLEFS : Lumière - symbole de Nicée - Grégoire de Nazianze - apophatisme patrologie.

Abstract. - Light as an Image of God and as a Name of Man in Gregory of Nazianzus. By Guillaume BADY.

In the second half of the IV ${ }^{\text {th }}$ century, Gregory of Nazianzus, later called «the Theologian », is one of the Greek Fathers whose works are abundantly infused with the theme of light. A preferred term for expressing divinity, light can make the connection between a positive formulation of God ( God is light») and a vision that is its «negative» counterpoint (God dazzles) : apophatic and cataphatic theologies are one and the same theology. In the anthropological field, the Incarnation of the Son, «Light of Light », gives a new meaning to a word which in Greek means «man »: the word phôs. By a kind of «photographic» revelation, Man finds in God his own identity as well as the path toward his deification.

KEYWORDS : Light - Nicene Creed - Gregory of Nazianzus - apophatism - patrology. 